\title{
BENCHMARKING OU ISOMORFISMO MIMÉTICO? UMA ANÁLISE À LUZ DA TEORIA DE DIMAGGIO E POWELL
}

\section{BENCHMARKING OR MIMÉTICO ISOMORPHISM? NA ANALYSIS IN THE LIGHT OF THE DIMAGGIO AND POWELL THEORY}

\begin{abstract}
Marcélia Aguiar Ferreira
Administradora, Especialista em Comunicação, Marketing e $\mathrm{RH}$, Coordenadora e Prof ${ }^{a}$. Titular do Curso de Administração da Universidade Presidente Antônio Carlos UNIPACTO MG-E-mail: marceliaaguiar@hotmail.com

Thiago Martins Monteiro Gondim

Psicólogo, e licenciatura em História, especializado em Saúde Mental, Estresse e Dependência Química, Professor na Faculdade Presidente Antônio Carlos de Teófilo Otoni, E-mail: thiagogondim2010@hotmail.com

\section{Adriana Aparecida da Conceição Santos Sá}

Doutoranda do Programa de Pós-graduação Interdisciplinar em Ciências Humanas da Universidade Federal de Santa Catarina (PPGICH/UFSC). Mestre em Gestão Integrada do Território pela Universidade Vale do Rio Doce - UNIVALE (2014). Especialista em Gestão de Micro e Pequenas Empresas pela Universidade Federal de Lavras - UFLA (2005). Professora Assistente da Universidade Federal dos Vales do Jequitinhonha e Mucuri (UFVJM - Campus do Mucuri) e-mail: admpublica@ead.ufvjm.edu.br
\end{abstract}

\section{Resumo}

O estudo possibilita ao leitor compreender a relação entre Benchmarking e isomorfismo mimético. A discussão preconiza a abordagem institucionallegitimidade que passa a ocupar com destaque as explicações dos fenômenos organizacionais. As estratégias de Benchmarking são amplamente abordadas atualmente. Tornou-se para as empresas uma excelente ferramenta de diferenciação. O cerne da questão é exatamente esse: "estratégia de diferenciação ou práticas hegemônicas de gestão". Para que se chegue ao objetivo do trabalho foi realizada pesquisa bibliográfica, que se baseou em livros, revistas, artigos, todo o assunto escrito acerca do tema Benchmarking e no que tange $O$ isomorfismo mimético, foi estudado o artigo de DiMaggio e Powell- A gaiola de Ferro revisitada: Isomorfismo Institucional e racionalidade coletiva nos campos organizacionais. A pesquisa nos possibilitou constatar a relação entre as teorias.

Palavras-chave: Benchmarking. Estratégia. Diferenciação.

\section{Abstract}


The study allows us to understand the relationship between Benchmarking and mimetic isomorphism. The discussion advocates the institutional approach legitimacy that comes to occupy with prominence the explanations of the organizational phenomena. Benchmarking strategies are widely covered today. It has become for companies an excellent tool of differentiation. The heart of the question is exactly this: "strategy of differentiation or hegemonic management practices". In order to arrive at the objective of the work, a bibliographical research was carried out, which was based on books, magazines, articles, the whole written subject on the subject Benchmarking and regarding the mimetic isomorphism, the article of DiMaggio and Powell- The cage of Iron Revisited: Institutional Isomorphism and Collective Rationality in Organizational Fields. The research allowed us to verify the relation between the theories.

Keywords: Benchmarking. Strategy. Differentiation.

\section{Introdução}

O mercado atual é caracterizado por intensa competição. Assim, a diferenciação é uma questão de sobrevivência para as empresas. Para se diferenciar entre as demais, a empresa necessita buscar obter vantagem competitiva. O benchmarking evidencia-se como excelente estratégia de vantagem competitiva, e está relacionado ao processo de melhoria contínua, e a disseminação do conhecimento na empresa. Segundo Camp (1995) benchmarking é a busca pelas melhorias práticas que conduzem uma empresa à maximização da performance empresarial. De forma generalizada, o benchmarking imita as ações de outras empresas, e procura fazê-lo de maneira aperfeiçoada, e com melhor desempenho e o isomorfismo mimético, segundo DiMaggio e Powell (2005) é resulta da padronização de respostas à incerteza do ambiente.

Considerando que o mercado é um universo de incerteza, percebe-se cada vez mais uma relevante homogeneidade de formas e práticas organizacionais segundo DiMaggio e Powell (2005). Essa incerteza do ambiente de competição faz com que as empresas tomem como modelo de gestão outros modelos, essa cópia constitui uma resposta à incerteza. Esses modelos podem ser definidos por acaso, ou por meio de consultorias ou associações. Diante do exposto, discorreremos no trabalho as ideias do isomorfismo mimético nas práticas de benchmarking, partindo do pressuposto da incerteza técnica e da estruturação isomórfica.

Conforme o estudo segue, este trabalho tem como objetivo responder a seguinte questão: Podemos considerar que as práticas de benchmarking é o isomorfismo mimético citado por DiMaggio e Powell? Para responder esta questão 
norteadora, temos os seguintes objetivos: Conhecer e analisar a relação entre isomorfismo mimético e Benchmarking.

\section{Referencial teórico}

\subsection{Benchmarking}

Araújo Júnior (2001, p. 241) afirma que "a origem do termo benchmarking é oriunda da agrimensura, em que é utilizado para definir um marco no terreno, com a finalidade de permitir comparações de altura, direção, distância, entre outros. "

Gariba Junior (2005, p.43) afirma que benchmarking é:

[...] um procedimento de pesquisa, contínuo e sistemático, pelo qual se realizam comparações entre organizações, objetos ou atividades, criandose um padrão de referencia. A técnica de benchmarking visa, portanto, à procura de pontos de referência que comparem o desempenho com a concorrência, com o objetivo de melhorar o rendimento naquele aspecto que se quer medir. $O$ benchmarking sugere um processo estruturado de identificação daquilo que se deseja aperfeiçoar, um processo de investigação de oportunidades de melhoria interna e um processo de aprendizagem, uma vez que não se trata de aplicar nada diretamente, mas sim adaptar as melhores práticas do processo à mentalidade e cultura da própria empresa.

Segundo Camp (1998 p.4) a Xerox "teve a sorte de descobrir e aplicar o benchmarking no início do seu esforço para combater a concorrência”.

Num tempo em que a dinâmica das relações empresariais vão passando por novos processos de atualizações e tomada de decisões, no entendimento de Maximiano (2004), benchmarking refere-se a técnica de comparação do desempenho de uma empresa com o de outra.

Segundo Brasil (2015), benchmarking é uma das mais significativas estratégias para melhorar a eficiência da empresa. Refere-se a um rigoroso processo de pesquisa que facilita aos gestores compararem produtos, técnicas empresariais, serviços e metodologias utilizadas pelos concorrentes, captando algumas características para alçarem um nível de superioridade gerencial ou operacional.

Araújo (2006 p.235) faz a seguinte afirmação sobre benchmarking:

É uma abordagem de gestão organizacional que conduz a tão desejada excelência, utilizando-se de procedimentos de investigação que pretendem 
reunir e adaptar as respostas encontradas por outras organizações. Longe de se igualar à mera cópia, revela alternativas valiosas de incrementos dos níveis de eficácia e eficiência.

Segundo Spendolini (1994), benchmarking é um processo ininterrupto de busca, que provê informações preciosas. Um processo de aprendizado com outro, que requer tempo e empenho, exigindo observância. Uma ferramenta viável que oferece informações utilizáveis para melhorar praticamente qualquer prática de negócio.

Neste contexto, Kearns (1998) afirma que benchmarking é o método contínuo de medição de produtos, serviços e práticas em relação aos mais fortes concorrentes ou em relação às empresas reconhecidas como líderes em seus segmentos.

\section{Tipos de benchmarking}

Camp (1998) entende que existem quatro tipos de benchmarking:

I) benchmarking com ações internas ou benchmarking interno; II) benchmarking com concorrentes externos ou competitivos; III) benchmarking com as melhores atividades externas ou os líderes da indústria (benchmarking funcional) e IV) o benchmarking de processos genéricos. Cada um tem seus benefícios e deficiências e, em certas condições, pode ser um mais apropriado que o outro.

Spendolini (1992), expressa que o benchmarking interno é a investigação da prática em departamentos distintos, buscando-se o melhor desempenho e discernindo-se as atividades de linha de linha de base e propulsores.

Segundo Zairi e Leonard (1995), o benchmarking com concorrentes diretos ou competitivos, pode ser utilizado como uma maneira de indicar às pessoas o seu desempenho contra a concorrência direta.

Cooke (1995), compreende que o benchmarking competitivo tem a finalidade de apontar a performance de outros concorrentes diretos. Entender as forças e as deficiências dos concorrentes é um passo significativo no delineado de uma estratégia bem sucedida.

Para Zairi e Leonard (1995), o benchmarking funcional defronta funções particulares com o melhor do setor e o melhor entre as organizações de porte símile.

Segundo Camp (1998), o benchmarking genérico é a forma mais fidedigna de benchmarking. Por meio dele, é possível o descobrimento de normas e métodos 
ainda não implementados na empresa do investigador. $O$ benchmarking genérico tem competência para evidenciar as melhores das melhores das práticas.

Segundo Brasil (2015), existem 4 tipos de benchmarking:

Benchmarking interno: procura pelas melhores práticas utilizadas dentro da própria empresa (filiais-modelo, departamentos que criam metodologias inovadoras, etc);

Benchmarking competitivo: a essência é a análise precisa das práticas da concorrência, visando superá-las. É difícil de ser efetivada, tendo em vista que as empresas não têm o hábito de "vazar" seus segredos tão facilmente aos concorrentes;

Benchmarking funcional: o que é comparado é o procedimento de trabalho entre as empresas, ainda que a comparação esteja sendo feita com empresas de segmentos diferentes;

Benchmarking de cooperação: duas empresas criam uma parceria, compartilhando informações de seus processos. Também acontece quando uma empresa "modelo" abre as portas de alguns processos para o aprendizado de outra. Isso pode ocorrer quando duas companhias têm distintos pontos de excelência ou quando uma dela permite o conhecimento de outra por razão de prestígio, notoriedade, etc.

\section{Isomorfismo mimético de Dimaggio e Powell}

A pergunta que introduz o texto a Gaiola de Ferro dos autores é: O que torna as organizações tão similares? Retrata os mecanismos de racionalização e burocratização, que se deslocou do mercado competitivo, para o Estado e para as categorias profissionais. Uma vez que um grupo de organizações emergem como um campo, surgindo assim um paradoxo: atores racionais tornam suas organizações cada vez mais similares, à medida que tentam transformá-las. Daí a ideia dos processos isomórficos: Coercitivo, Mimético e Normativo. Nesse estudo trataremos apenas do mimético.

Segundo DiMaggio: com o domínio do Estado e de outras grandes organizações, as organizações se tornam cada vez mais homogêneas, pois as estruturas se refletem cada vez mais regras institucionalizadas e legitimadas pelo Estado e dentro do Estado. 
Os autores retratam que, no caso das estratégias organizacionais, primeiro, a emergência e a estruturação de um campo organizacional como resultado de atividades de um grupo diverso de organizações e, da mesma forma, dos ingressantes, uma vez que o campo seja estabelecido.

Trazem ainda, a ideia da incerteza que constitui o isomorfismo mimético, essa incerteza justifica a imitação. Quando as tecnologias organizacionais são insuficientemente compreendidas, quando as metas são ambíguas ou o ambiente cria incerteza simbólica, as organizações podem vir a tomar outras organizações como modelo. As vantagens do comportamento mimético, em termos de economia de ações humanas, são consideráveis.

Tomar outras organizações como modelo, como denominamos, constitui resposta à incerteza. Os modelos podem ser definidos involuntariamente, indiretamente por meio de transferência ou rotatividade de funcionários, ou por outras organizações como consultoria ou associações. Até mesmo as inovações podem ser creditadas ao fato de possuir outras organizações como modelo.

Alchian (1950) observou que enquanto há aquelas que inovam conscientemente, há aquelas que, em suas tentativas imperfeitas de imitar as outras, inovam inconscientemente por meio da aquisição involuntária de atributos únicos e inesperados ou não procurados, os quais, sob circunstância correntes, se promovem parcialmente responsáveis pelo sucesso. Outros, por sua vez, procurarão copiar singularidades e, o processo de inovação- imitação prossegue.

(Dimaggio e Powell, 1991, p.44). Percebe-se então, que as organizações se tornam isomórficas ao seu meio por "pressões Formais ou influencias exercidas nas organizações por outras organizações [ou] imposição de procedimento operacionais padronizados". Dimaggio e Powell (1991). Sendo assim, Dimaggio traz a metáfora "jaula de ferro" para elucidar o comportamento organizacional.

\section{Metodologia}

A pesquisa desenvolvida, quanto à tipologia, trata-se de pesquisa bibliográfica. Pois, segundo Marconi e Lakatos (1992), a pesquisa bibliográfica é o levantamento de cada bibliografia já publicada, na forma de livros, revistas, publicações avulsas e imprensa escrita. O seu propósito é fazer com que o pesquisador entre em familiaridade com todo o material escrito sobre determinado assunto. Ela pode ser encarada como o primeiro passo de toda a pesquisa científica. 
Conforme esclarece Boccato (2006, p. 266):

A pesquisa bibliográfica busca a resolução de um problema (hipótese) por meio de referenciais teóricos publicados, analisando e discutindo as várias contribuições científicas. Esse tipo de pesquisa trará subsídios para o conhecimento sobre o que foi pesquisado, como e sob que enfoque e/ou perspectivas foi tratado o assunto apresentado na literatura científica. Para tanto, é de suma importância que o pesquisador realize um planejamento sistemático do processo de pesquisa, compreendendo desde a definição temática, passando pela construção lógica do trabalho até a decisão da sua forma de comunicação e divulgação.

\section{Considerações finais}

É evidente que o mercado atual está mais competitivo, as empresas procuram cada vez mais se destacar, diferenciar-se tornou-se essencial à sobrevivência da empresa.

Este artigo objetivou compreender a relação entre Benchmarking e isomorfismo mimético. A discussão preconiza a abordagem institucionallegitimidade que passa a ocupar com destaque as explicações dos fenômenos organizacionais. As estratégias de Benchmarking são amplamente abordadas atualmente.

Concluímos então, a analogia entre benchmarking e isomorfismo mimético. Podemos sim considerar que as práticas do primeiro, corresponde à definição do segundo. Compreendemos tal relação no decorrer das definições dos termos e assim entendendo tal similaridade. As organizações tendem a tomar como modelo em seu campo outras organizações que elas percebem ser mais legitimas ou bemsucedidas. Segundo DiMaggio e Powell à ubiquidade de determinados tipos de arranjos estruturais podem ser mais provavelmente creditadas à universalidade de processos miméticos do que a concreta evidencia de que modelos adotados aumentam a eficiência.

Entendemos como válido o uso do processo de benchmarking nas organizações, principalmente por aquelas que estão iniciando suas atividades no mercado. Porém é preciso ter cuidado em "copiar" tais processos, pois os mesmos podem não ser viáveis na sua empresa.

\section{Referências}


ANDREWS, K. R. The concept of corporate strategy. In: The strategy process: concepts and contexts. Prentice Hall, 1980.

ARAÚJO JÚNIOR, Rogério Henrique de. Benchmarking. In: TARAPANOFF, Kira (Org.). Inteligência organizacional e competitiva. Brasília: EDUNB, 2001. p. 241 263

ARAÚJO, Luiz César G. Gestão de Pessoas - Estratégias e Integração Organizacional. São Paulo: Atlas, 2006.

ASCENSÃO, Carlos. Implementação do Benchmarking. Disponível em: http://www.portalwebmarketing.com/Gest\%C3\%A3o/lmplementa\%C3\%A7\%C3\%A30 doBenchmarking/tabid/1176/Default.aspx > Acesso em 31 de out. de 2016.

BALLOU, R.H. Gerenciamento da Cadeia de Suprimentos/ Logística Empresarial. 5a . Edição. Porto Alegre: Bookman, 2006.

BOCCATO, V. R. C. Metodologia da pesquisa bibliográfica na área odontológica e o artigo científico como forma de comunicação. Rev. Odontol. Univ. Cidade São Paulo, São Paulo, v. 18, n. 3, p. 265-274, 2006.

BRASIL, Endeavor. Uma espiada na grama do vizinho, ou: como fazer benchmarking. Disponível em <https://endeavor.org.br/benchmarking/> Acesso em 31 de out. de 2016.

CAMP, Robert C...Benchmarking: the search for industry best practices that lead to superior performance.ASQC Quality Press, 1989.

CAMP, R. C. Benchmarking: identificando, analisando e adaptando as melhores práticas da administração que levam à maximização da performance empresarial: o caminho da qualidade total. 2.ed. São Paulo: Pioneira, 1995.

CAMP, Roberto C. Benchmarking: o caminho da qualidade total. 3. ed. São Paulo: Pioneira, 1998.

CHIAVENATO, I.; SAPIRO, A. Planejamento estratégico: fundamentos e aplicações. Rio de Janeiro: Companhia de Vídeo, Campus 2004.

CHIAVENATO, Idalberto. Introdução à Teoria Geral da Administração: na administração das organizações. Edição Compacta. $3^{\circ}$ Ed. Rio de Janeiro: Elsevier, 2004.

CHIAVENATO, Idalberto; Teoria Geral da Administração. 3a ed, São Paulo: McGraw-Hill, Ltda, 1987.

COOKE, A. Benchmarking 101. San Francisco Jessey Bars, 1995.

DESSLER, Gary. Administração de Recursos Humanos. 2. ed. São Paulo: Prentice Hall, 2003. 
FISCHMANN, Adalberto Américo; ALMEIDA, Martinho Isnard Ribeiro de. Planejamento estratégico na prática. 2 ed. São Paulo: Atlas, 1991.

FREZATTI, Fabio. Orçamento empresarial: planejamento e controle gerencial. 5 . ed. São Paulo: Atlas, 2009.

GARIBA JUNIOR, M. Um modelo de avaliação de cursos superiores de tecnologia baseado na ferramenta Benchmarking. Tese Doutorado em Engenharia de Produção, Universidade Federal de Santa Catarina, Florianópolis, 2005.

KEARNS, D. Quality improvement begins at the top. Ed World 20, 1998.

KOTLER, Philip. Administração de Marketing. São Paulo: Ed. Atlas, 1994.

KOTLER, Philip. Administração de marketing: análise, planejamento, implementação e controle. 2. ed. São Paulo: Editora Atlas, 1992.

LACOMBE, Francisco. Teoria geral da administração. Saraiva, 2009.

MARCONI, Marina de Andrade; LAKATOS, Eva Maria. Metodologia do trabalho científico. São Paulo: Editora Atlas, 1992. 4a ed. p.43 e 44.

MAXIMIANO, A. C. A. Introdução à administração. 6. ed. São Paulo: Atlas, 2004.

MAXIMIANO, Antonio Cesar A. Introdução à Administração. 7. ed. São Paulo: Atlas, 2009.

OLIVEIRA, D.P.R. de. Planejamento estratégico: conceitos, metodologia e práticas. 22. Ed. São Paulo: Atlas, 2006.

OLIVEIRA, Djalma de Pinho Rebouças; Planejamento Estratégico. 26ª ed, São Paulo: Atlas, 2009.

ORLICKAS, Elizen da. Modelos de gestão: das teorias da administração à gestão estratégica. São Paulo: IBPEX, 2010.

PORTER, M. E. Vantagem competitiva. Trad. Elizabeth M. P. Braga. RJ: Campus, 1990a.

PORTER, M. E.; Competitive advantage. New York: Free Press. 1985.

PORTER, M. E.; Competitive strategy. New York: The Free Press. 1980.

PORTER, Michael E. Estratégia Competitiva - Técnicas para análise de indústrias e da concorrência. 18ª Edição. São Paulo-SP: Campus, 1986.

PORTER, Michael. Vantagem competitiva: criando e sustentando um desempenho superior. 16 ed. Rio de Janeiro: Campus, 1989. 
SAMPAIO, Carlos Alberto Cioce. Gestão organizacional estratégica para o desenvolvimento sustentável. Itajaí: Editora da Univale, 2000.

SPENDOLINI, M. J. Benchmarking. São Paulo: Makron Books, 1994.

SPENDOLINI, Michael J. Benchmarking. São Paulo: Makron Books, 1992.

THOMPSON, Arthur A. Estratégia e vantagem competitiva. In:

Planejamento estratégico. São Paulo: Pioneira, 2000. cap.5. p. 15 p. 153-196.

ZAIRI, M; LEONARD, P. Benchmarking prático - o guia completo. São Paulo: Atlas, 1995. 